\title{
INS-AIDED 3D LIDAR SEAMLESS MAPPING IN CHALLENGING ENVIRONMENT FOR FUTURE HIGH DEFINITION MAP
}

\author{
G. J. Tsai ${ }^{1}$, K. W. Chiang ${ }^{1}$, N. El-Sheimy ${ }^{2}$ \\ ${ }^{1}$ Dept. of Geomatics Engineering, National Cheng-Kung University, No. 1, Daxue Road, East District, Tainan, Taiwan \\ - tpp1114@gmail.com \\ ${ }^{2}$ Dept. of Geomatics Engineering, University of Calgary, 2500 University Dr NW, Calgary, AB T2N 1N4, Canada \\ - elsheimy@ucalgary.ca
}

KEY WORDS: INS/GNSS/LiDAR, Simultaneously Localization and Mapping, high definition map

\begin{abstract}
:
With advances in computing and sensor technologies, onboard systems can deal with a large amount of data and achieve real-time process continuously and accurately. In order to further enhance the performance of positioning, high definition map (HD map) is one of the game changers for future autonomous driving. Instead of directly using Inertial Navigation System and Global Navigation Satellite System (INS/GNSS) navigation solutions to conduct the Direct Geo-referencing (DG) and acquiring 3D mapping information, Simultaneous Localization and Mapping (SLAM) relies heavily on environmental features to derive the position and attitude as well as conducting the mapping at the same time. In this research, the new structure is proposed to integrate the INS/GNSS into LiDAR Odometry and Mapping (LOAM) algorithm and enhance the mapping performance. The first contribution is using the INS/GNSS to provide the short-term relative position information for the mapping process when the LiDAR odometry process is failed. The checking process is built to detect the divergence of LiDAR odometry process based on the residual from correspondences of features and innovation sequence of INS/GNSS. More importantly, by integrating with INS/GNSS, the whole global map is located in the standard global coordinate system (WGS84) which can be shared and employed easily and seamlessly. In this research, the designed land vehicle platform includes commercial INS/GNSS integrated product as a reference, relatively low-cost and lower grade INS system and Velodyne LiDAR with 16 laser channels, respectively. The field test is conducted from outdoor to the indoor underground parking lot and the final solution using the proposed method has a significant improvement as well as building a more accurate and reliable map for future use.
\end{abstract}

\section{INTRODUCTION}

To further enhance the performance of positioning, high definition map (HD map) is one of the game changers for future autonomous driving. Compared with the traditional 2 dimensional (2D) map, HD map expands one dimension in the vertical direction to make sure the vehicle itself locates in the correct and safe place (Ma, 2017). For example, HD map should allow the autonomous systems to recognize either the vehicle is on the highway or under the highway, on the road or in the underground parking lot. This map also needs to be generated in the global coordinate system to share consistent information with other vehicles. In addition, the accuracy requirement is relatively higher than the traditional map. In order to help the self-driving car successfully and safely arrive at the destination, all of the features or the geospatial information should have the sub-meter accuracy (Farrell et al., 2016). However, it is not easy to generate 3D maps corresponding to the sub-meter accuracy, particularly in Global Navigation Satellite System (GNSS) signal outage environment.

LiDAR is an essential component when it comes to HD map. LiDAR has become more and more popular these days. With the advanced optical technology and hardware design, it becomes an integral part not only in surveying but also any field which is related to geospatial information. LiDAR is a cost-effective system to collect the geospatial information, allowing the 3D spatial information of objects to be calculated and measured. However, the information acquired from LiDAR is not enough for most applications, which needs to combine with other sensors for those mobile laser scanning systems (MLS). MLS can be employed in dynamic environments and are capable of being adopted in traffic-related applications, such as the collection of road network databases, inventory of traffic sign and surface conditions (Puente et al., 2012). Further applications of generated point cloud data have been used in HD map and multi-scanner self-calibration (Puente et al., 2012; Seif and Hu, 2016).

Recently, increasing numbers of researches have concentrated on self-driving applications. MLS fuses the hardware and software of positioning and orientation systems (POS) and mapping sensors to directly determine the position of point of interest (POI) remotely. POS includes typically an inertial navigation system (INS) and GNSS. GNSS gives the absolute position and velocity by using pseudo-range and carrier-phase measurement from the satellite signal. INS is a self-contained navigation system which is able to continuously track the position and orientation of a vehicle from a known initial point without any external signal (Titterton et al., 2004). Both navigation systems have their own advantages and disadvantages. Therefore, INS/GNSS has become one of the most popular integrated positioning systems. Researchers introduced the INS/GNSS into MLS to acquire the continuous exterior orientation parameters (EOPs). The integrated POS overcomes the drawbacks by only using an integrated system and continuously provides stable navigation information. This kind of systems combining with the other mapping sensors can meet the need for rapidly collecting geospatial data by using the direct geo-referencing (DG) mathematical model.

However, MLS is contaminated by several error sources, such as GNSS time error, time synchronization between GNSS, INS, and a laser scanner, interpolation of INS/GNSS measurements, system components mounting error, laser range and encoder angle error, etc. (Baltsavias, 1999; Katzenbeisser, 2003; Schenk, 2001). In GNSS-denied environment, the navigation performance of INS-only drops quickly over time in accordance with the grade of the IMU itself. In order words, it is difficult to 
achieve the sub-meter accuracy of HD map in an indoor environment if only using INS/GNSS integrated system.

Simultaneously localization and mapping (SLAM), on the other hand, relies more heavily on environmental features to derive the position and attitude of the robot itself. With the increasing computational power capabilities of contemporary computers, perception sensors have begun to be adopted as a component in the positioning methods. LiDAR-SLAM has been widely discussed and improved over the last decade. 2D LiDAR- SLAM reduces the point cloud information and re-projects the environment into 2D plane. Occupancy grid SLAM is one of the famous SLAM methods that transforms the 2D point cloud into an occupancy grid map. Gmapping and Hector SLAM are all based on the occupancy grid map. Gmapping utilizes RaoBlackwellized particle filters (RBPFs) to estimate the real-time position and conduct the mapping at the same time (Grisetti et al., 2007). Hector SLAM adopts scan-matching approach according to the Gauss-Newton model which increases the flexibility and adaptability for different applications (Kohlbrecher et al., 2011). To meet the demand of HD map, 3D information is necessary. One of the 6-DOF LiDAR-SLAM registration methods is iterative closest point (ICP) that registers the closest points together without considering the feature information (Holz et al., 2015). However, ICP is time-consuming and the accuracy depends on the density of point cloud. Feature-based, on the contrary, is relatively faster and robust. Features such as edge reflectivity are used to generate as a grid representation and can be matched with the prior map (Castorena and Agarwal, 2017). Hata and Wolf proposed the road marking detection based on the Otsu thresholding method and Monte Carlo localization method (Hata and Wolf, 2015). Feature-based LiDAR-SLAM such as LiDAR odometry and mapping (LOAM) extracts the features in a consecutive point cloud (Zhang and Singh, 2017).

With the aiding from the prior map information, the accuracy of the LiDAR-SLAM can achieve the precise positioning demand and meet the requirement of sub-meter accuracy (Levinson et al., 2007). However, how to generate accurate map information in GNSS-denied environment is still a great challenge over the decades. The results from pure LiDAR-SLAM only present the error of overall loop closure and are always overestimated because of the loop closure detection. This paper develops the INS-aiding 3D LiDAR-SLAM based on the LiDAR odometry and mapping method. By using reliable information from INS/GNSS and proposed failure detection, the results can be further improved compared with the pure SLAM solution.

\section{METHODOLOGY}

This research proposes the fusion algorithm to integrate INS/GNSS solution with LiDAR SLAM. Figure 1 shows the flowchart of data processing. As can be seen from this figure, INS/GNSS can be considered as the external source to improve the SLAM. First of all, the IMU and GNSS data is integrated using the proposed EKF as well as adding motion constraints to further enhance the navigation performance. After the integration, the filtered solution including position, velocity, and attitude will feed into the LiDAR SLAM algorithm.

There are two major processes in LOAM, LiDAR odometry and LiDAR mapping. In the odometry process, two consecutive point cloud sets will be registered based on the detected features, such as edge and planar points. The main cost function is formulated by the distance between correspondences of edge and planar points and can be determined by Levenberg-Marquardt (LM) method in real-time. In order to make the global consistency and loop closuring, the mapping process is to register the point cloud into the global map with the same cost function but extracting the correspondences from the global map.

To address these divergence and drift issues in LOAM, the proposed integration takes the INS/GNSS into account, utilizing the advantages to compensate and enhance the overall performance of SLAM. The error of INS can also be restricted using the zero velocity update (ZUPT) and non-holonomic constraint (NHC) which guaranteed the short-term relative position of INS is able to benefit the mapping process even without using odometry process. The proposed INS-aiding strategy not only take the INS/GNSS solution as the initial value but also uses it to detect the outlier solution in SLAM. The following sections will provide more details.

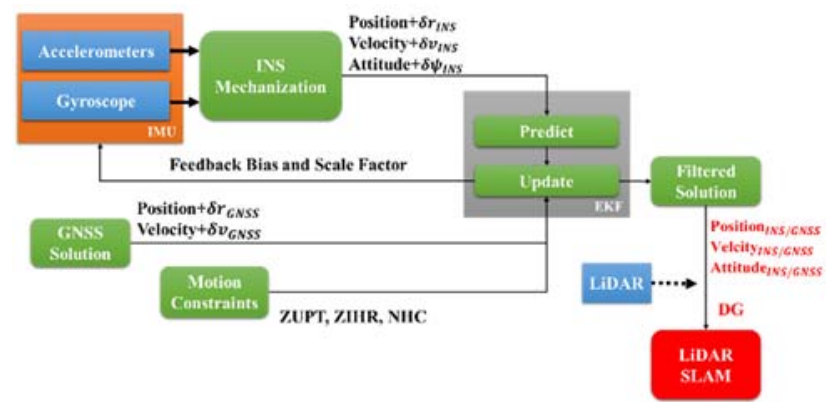

Figure 1. The flow chart of data processing

\subsection{INS/GNSS Integration}

INS/GNSS integration has become the most commonly used model for navigation systems. The fundamental method relies on integrating two sets of data using EKF. The complementary error characteristics make INS/GNSS integrated system more robustness. However, problems like drift during GNSS signal outages and multipath effect in urban areas are common. To address these issues, the proposed integrated algorithm adopts the vehicle motion constraints to control the drift problem, particularly in GNSS-denied environment.

Kalman filter extends the concept of least-squares to include the knowledge of how the states vary in time. In other words, KF predicts the behavior of states and estimates the covariance matrix. Generally, KF derives the optimal states by minimizing the covariance of the estimation error, and the recursive form benefits to efficient implementation. These characteristics make $\mathrm{KF}$ being widely used in optimal time-varying estimation, especially for INS/GNSS integrated navigation (Aggarwal et al., 2010; Farrell, 2008; Gebre-Egziabher and Gleason, 2009).

The developed INS/GNSS scheme uses the implementation in (Shin, 2005). The states of the proposed EKF is defined as:

$$
\begin{gathered}
x_{k}=\left[\begin{array}{ccccc}
r & v & \psi & b_{a} & b_{g}
\end{array}\right]_{15 \times 1}^{T} \\
\hat{x}_{k}=x_{k}+\delta x_{\mathrm{k}}
\end{gathered}
$$

where $x$ is the state vector, $\hat{x}$ is the updated states, $\delta x$ is the error of state vector, and subscript $k$ is the parameter at epoch $k . r, v$ and $\psi$ are the main navigation information, representing position, velocity and attitude, respectively; $b_{a}$ and $b_{g}$ are biases of the accelerometers and gyroscopes respectively.

An INS system model is implemented as the discrete-time form and is represented as: 


$$
\delta x_{k+1}=\Phi_{k, k+1} \delta x_{k}+\omega_{k}
$$

The measurement model of EKF is implemented in discrete-time form as follows:

$$
\delta z_{k}=\mathrm{H}_{k} \delta x_{k}+\epsilon_{k}
$$

where $\Phi_{k, k+1}$ represents the state transition matrix and $\omega_{k}$ represents the white noise sequence of the system. $\mathrm{H}_{k}$ is the design matrix necessary to project the states into measurements, and $\epsilon_{k}$ is the white noise sequence of the measurement.

In GNSS-denied environment, INS-only suffers from the rapid error accumulation. To mitigate this impact, vehicle information combined with the physical behavior of the automobile plays an important role. NHC is to constraint the lateral and vertical velocity of vehicle based on the physical behavior of moving land vehicles (Shin, 2005). In addition to NHC, the stationary is also one of the useful information for controlling the drifting error. ZUPT and zero integrated heading rate (ZIHR) are the constraints on velocity and attitude domains. If the system detects the stationary, the velocity and the heading change rate will be limited to zero, which can reset the velocity error and suppress the drifting error in position and attitude domains.

\subsection{Direct Geo-reference}

In order to combine the EOPs information with other sensors, DG mathematical model describes the relationship of each sensor and benefits the integration system to rapidly collect geospatial data. In other words, DG allows us to directly acquire the objects' location by integrating different measurements together. With regard to the point cloud data processing, DG combines the INS/GNSS with the LiDAR system to produce a geo-referenced point clouds.

As shown in Figure 2, this paper uses the land vehicle as the major platform to collect the data. The geo-referencing formula is written as:

$$
r_{i}^{m}=r_{n a v}^{m}(t)+R_{b}^{m}(t) \times\left(R_{l}^{b} p_{i}^{l}+r_{i l}^{b}\right)
$$

where $r_{i}^{m}$ is the coordinate vector of $\mathrm{i}$-th laser point in the $\mathrm{m}$ frame; $r_{\text {nav }}^{m}(t)$ is the position vector at time $t$ of the INS/GNSS in the mapping-frame (m-frame); $R_{b}^{m}(t)$ is the rotation matrix between the navigation system $\mathrm{b}$-frame and the $\mathrm{m}$-frame; $R_{l}^{b}$ is the differential rotation matrix between the LiDAR-frame (1frame) and the body-frame (b-frame), determined by calibration; $p_{i}^{l}$ is the coordinate vector of $\mathrm{i}$-th object point in the l-frame; $r_{i l}^{b}$ is the vector between INS centre and LiDAR, determined by calibration.

In l-frame, the object point $p_{i}^{l}$ is written as follows:

$$
p_{i}^{l}=\left[\begin{array}{c}
X_{i}^{l} \\
Y_{i}^{l} \\
Z_{i}^{l}
\end{array}\right]=\left[\begin{array}{c}
D * \cos (\omega) * \sin (\alpha) \\
D * \cos (\omega) * \cos (\alpha) \\
D * \sin (\omega)
\end{array}\right]
$$

where $X_{i}^{l}, Y_{i}^{l}$, and $Z_{i}^{l}$ are the point coordinates in the $l$-frame; $D$ is the distance between the object and LiDAR center; $\omega$ is the vertical angle as indicated by the laser channel, and $\alpha$ is the horizontal angle between the $y^{l}$-axis and object.

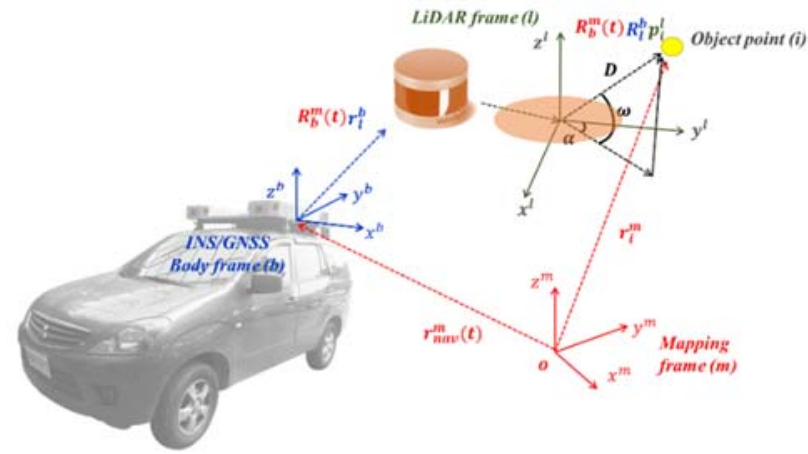

Figure 2. Direct geo-referencing model

\subsection{INS-AIDED 3D LiDAR SLAM}

In this paper, the INS-aided 3D LiDAR-SLAM can be divided into LiDAR odometry and LiDAR mapping. Before LiDAR odometry and LiDAR mapping, the feature extraction is used to find the corresponding feature in each scan (the total point cloud measurements within 360 degrees horizontal rotation). The concept of these two processes is based the LOAM (Zhang and Singh, 2017).

As shown in Figure 3, LiDAR odometry plays the basic positioning estimation model to continuously $(10 \mathrm{~Hz})$ calculate the relative position and attitude with respect to the previous scan. However, it is inevitable to accumulate the error for relative positioning technique. In order to mitigate the error accumulation and improve the consistency of the map itself, LiDAR mapping is proposed acting as the refined model $(5 \mathrm{~Hz})$ to correct the drifting error. LiDAR mapping registers the features extracted from the current scan to global map where the whole features are stored in. In order words, instead of only aligning with the previous scan, LiDAR mapping takes the whole nearest features into account. Furthermore, it is important to define the geometric features and how to extract them from the messy and heavy point clouds. Moreover, this paper proposes the INS-aided concept in SLAM, failure detection, to detect the failure solution LiDAR odometry and further improve the solution in the mapping process.

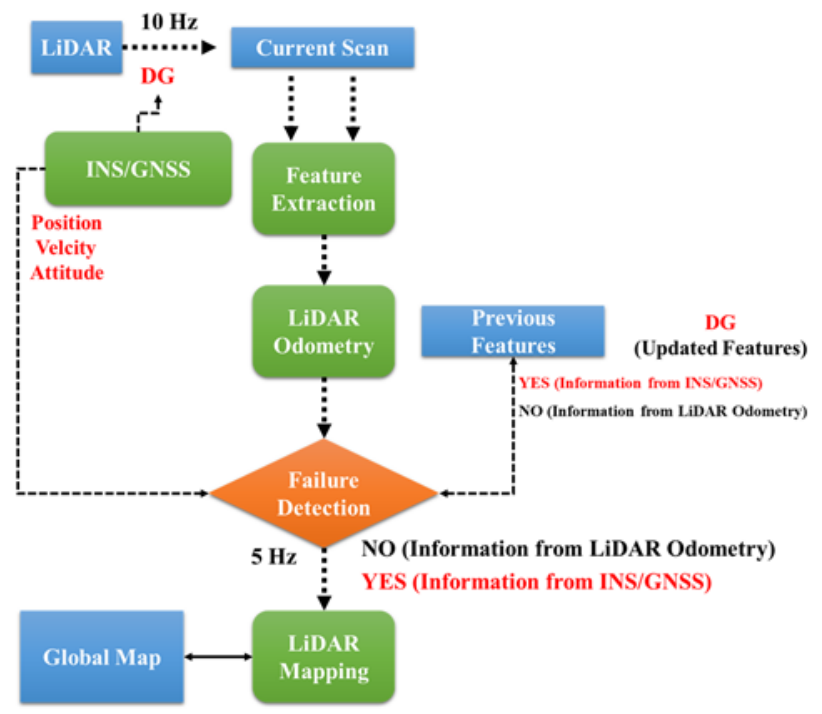

Figure 3. SLAM-aiding INS/GNSS integration 
2.3.1 Feature Extraction: In the feature extraction process, the edge point and planar surface are extracted with co-planar geometric relationship from individual channels. In terms of the Velodyne LiDAR used in this paper, there are 16 channels for the individual scan. Each channel is equally sized scan regions and divided into 6 sub-regions $(S)$ for evenly distributing the features within the environment. The definition of curvature $(c)$ to determine the edge and planar points is written as follows:

$$
c=\sum_{j \in S, j \neq i}^{n}\left(p_{k, i}^{m}-p_{k, j}^{m}\right)^{2}
$$

where $c$ is the curvature to evaluate the smoothness of the local surface, $n$ is the total number of points in the sub-region, $i$ is a point in the sub-region and $j$ is the consecutive point of $i$-th point

The curvature of each point is sorted according to its value. If the curvature is higher than the preset threshold, it is determined as the edge point and vice versa. In addition, there are two cases might lead to unreliable extraction as shown in Figure 4. The first case occurs when the point is extracted from the object edge line where is roughly parallel with the laser beam. The second case is to select the feature on the boundary of an occluded region. In this situation, it is clear to identify that the selected point is not the edge point and it is connected with other surfaces. It is because the laser beams are blocked by another object. Once the LiDAR moves to the left-hand side, the occluded part will be observable and will not be considered as the edge point again.

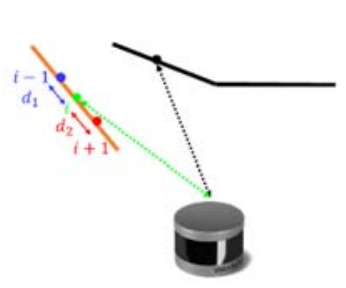

(a) Roughly parallel

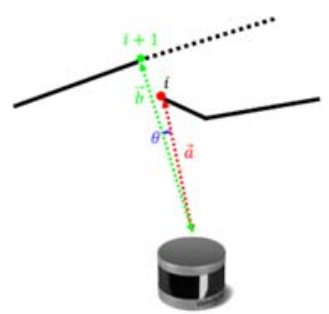

(b) Occluded
Figure 4. Unreliable extraction from two cases

2.3.2 LiDAR Odometry: In LiDAR odometry, the first step is to find the corresponding feature in every scan. Based on the geometric relationship between each correspondence, the objective function can be built afterward. The relative position and attitude are estimated by taking account whole geometric relationship in the objective function. Therefore, LiDAR odometry can be separated into three parts, finding correspondence, formulating the objective function and motion estimation.

First, two geometries are utilized, edge line as the correspondence for an edge point and planar surface as the correspondence for a planar point. The edge line is composed of two edge points $\left(j_{k-1}\right.$ and $\left.l_{k-1}\right)$ from the previous scan $(k-1)$. These two edge points are the closest points to the current edge point $\left(i_{k}\right)$ and must be selected in different channels. This is to prevent the selected edge line located on the same surface where is parallel with the same scanning channels. The corresponding planar surface can be selected based on the three closest neighbors $\left(j_{k-1}, l_{k-1}\right.$ and $\left.h_{k-1}\right)$ of current planar feature $\left(i_{k}\right)$.

Second, the objective functions are built based on these two geometries. For the edge point $\left(i_{k}\right)$, it is assumed to align with the determined edge line, which means the distance between the edge line and edge point should be zero. The point-to-line distance is defined as:

$$
\operatorname{dist}_{\text {line }}=\frac{\left\|\left(\tilde{p}_{k, i}-p_{k-1, j}\right) \times\left(\tilde{p}_{k, i}-p_{k-1, l}\right)\right\|}{\left\|p_{k-1, j}-p_{k-1, l}\right\|}
$$

where dist $_{\text {line }}$ is the point-to-line distance distance, $p$ represents the coordinates of point, $\times$ means cross product. In this equation, it can be interpreted that the area of the parallelogram is equal to the base multiplies the height.

Similar concept from the point-to-line distance, the point-toplane distance is defined as:

$$
\text { dist }_{\text {plane }}=\frac{\left\|\left(\tilde{p}_{k, i}-p_{k-1, l}\right) \cdot\left(p_{k-1, j}-p_{k-1, l}\right) \times\left(p_{k-1, j}-p_{k-1, h}\right)\right\|}{\left\|\left(p_{k-1, j}-p_{k-1, l}\right) \times\left(p_{k-1, j}-p_{k-1, h}\right)\right\|}
$$

where dist $_{\text {plane }}$ is the point-to-plan distance distance, $\cdot$ means dot product. In this equation, it can be considered as volume of a parallelepiped by the multiplication of the base area and the height.

The final part of LiDAR odometry is to estimate the relative position and attitude. In this SLAM algorithm, the motion of sensor is assumed to be constant angular velocities and linear velocities. As a result, the rigid transformation is written as:

$$
\begin{aligned}
& p_{k, i}=R_{k-1}^{k} \times \tilde{p}_{k, i}+r_{k-1}^{k} \\
& f\left(\tilde{p}_{k, i}, R_{k-1}^{k}, r_{k-1}^{k}\right)=d
\end{aligned}
$$

where $p_{k, i}$ and $\tilde{p}_{k, i}$ are the $\mathrm{i}$-th coordinates of feature points after and before transformation, $R_{k-1}^{k}$ is the rotation matrix from time $k-1$ to $k$ and $r_{k-1}^{k}$ is the translation vector.

In order to solve the nonlinear function $(f)$, the LM algorithm is used (Hartley and Zisserman, 2003). The LM is considered as the combination between Gauss-Newton algorithm and the method of gradient descent especially addressing the nonlinear least square problems.

Consequentially, edge and planar features are transformed and stored for the next registration. Since the DG is conducted ahead, the transformation parameters from the LiDAR odometry can be further improved by matching features.

2.3.3 LiDAR Mapping: LiDAR odometry is a process to register features in the current scan to the previous features. In addition, the transformation during each scan is assumed to be the constant and linear movement. However, this assumption is not always appropriate especially applied in the speedy land vehicle. The LiDAR mapping plays the primary role to correct the distorted movement from the LiDAR odometry as well as generating the consistent global map for each LiDAR mapping registration.

The concept of the LiDAR mapping is to align the features point with the global map where is also the incremental map based on each LiDAR mapping registration. LiDAR mapping extracts the 
corresponding points from the global map and matches these points with the features from LiDAR odometry. Afterward, these features will also be stored in the global map which gradually expands the map in order to enhance the future loop closure solution. However, it is time-consuming to conduct this global registration process. In this structure, the LiDAR mapping is processed in the lower frequency, $5 \mathrm{~Hz}$, while LiDAR odometry is $10 \mathrm{~Hz}$.

2.3.4 Failure Detection: In the proposed integration scheme, failure detection is adopted to detect the unreasonable solution from the LiDAR odometry. By continuously updating the navigation solution and feedback bias for INS, it is assumed that INS is capable of monitoring other outlier measurements in a short period of time. According to this assumption, the proposed fusion algorithm uses the INS/GNSS velocity to detect whether the LiDAR odometry process works properly or not. In addition, the proposed fusion algorithm also takes distances of the final corresponding points (edge and planar points) into account. This is to evaluate the performance of LiDAR SLAM itself. Thus, there are two conditions to evaluate the performance of LiDAR odometry.

$$
\left\{\begin{array}{c}
\text { if } \frac{\sum_{i}^{n} f\left(p_{k, i}, R_{k-1}^{k}, r_{k-1}^{k}\right)}{n} \leq \theta_{\text {corDist }}, \text { is qualified. } \\
\text { if } \Delta \text { Velocity } \leq \theta_{\text {velocity }}
\end{array}\right.
$$

The corresponding distance including point-to-line and point-toplane should smaller than the preset threshold $\left(\theta_{\text {corDist }}\right)$ after the LM algorithm. Specifically, the average residual error should be less than a certain amount of value. In addition, the offset between the SLAM-derived velocity and INS/GNSS velocity has to be less than the $\theta_{\text {velocity }}$.

LiDAR odometry is based on the dead reckoning (DR) for the SLAM algorithm. Hence, if there is an outlier solution estimated from the LiDAR odometry, the error will accumulate until the next mapping process. However, LiDAR odometry will provide the unreliable initial solution to LiDAR mapping which means the mapping process also has great possibilities of suffering from the same problem.

In proposed failure detection shown in Figure 3, once the result from LiDAR odometry is detected as an unreliable solution, there are two strategies to address. Since LiDAR odometry is a continuous process to align the features from the current scan with the previous scan. If LiDAR odometry performs unstably, the first strategy is to replace the LiDAR odometry solution with the INS/GNSS solution. By using the more stable INS/GNSS solution, it prevents the outlier from influencing the updating process which means the edge and planar points are correctly transformed and stored for the next aligning. The second strategy in odometry process benefits the LiDAR mapping, the INS/GNSS provides initial value $\left(R_{k-4}^{k}, r_{k-4}^{k}\right)$ for the mapping process instead of using unreliable odometry solution. This strategy makes sure the mapping process has a relatively trustable initial value to conduct the LM algorithm.

\section{EXPERIMENT}

This paper uses the high-grade commercial product, SPAN-LCI, as the reference POS. For INS-aided implementation, lower grade INS (C-MIGITS) with low-cost LiDAR (VLP-16) are used. To evaluate the performance fairly, this paper uses the same differential GNSS (DGNSS) solutions from the commercial product SPAN-LCI receiver. In addition, the initial value of pure SLAM is also from the reference. Figure 5 shows the platform we used in this paper, Table 1 and Table 2 give the specification of two POSs as well as LiDAR sensor.

\begin{tabular}{ccc}
\hline \hline & \multicolumn{2}{c}{ SPAN-LCI } \\
\cline { 2 - 3 } & Accelerometer & Gyroscope \\
\hline Bias Instability & $\leq 100 \mu \mathrm{g}$ & $\leq 0.05^{\circ} / \mathrm{hr}$ \\
Random Walk & $\leq 100 \mu \mathrm{g}$ & $\leq 0.012 \% / \sqrt{\mathrm{hr}}$ \\
Noise & $/ \sqrt{\mathrm{Hz}}$ & $\leq$ \\
\hline
\end{tabular}

\begin{tabular}{ccc}
\hline & \multicolumn{2}{c}{ C-MIGITS ${ }^{\circ}$ III } \\
\cline { 2 - 3 } & Accelerometer & Gyroscope \\
\hline Bias Instability & $200 \mu \mathrm{g}$ & $1^{\circ}-3 \% / h r$ \\
$\begin{array}{c}\text { Random Walk } \\
\text { Noise }\end{array}$ & $60 \mu \mathrm{g} / \sqrt{\mathrm{Hz}}$ & $0.035^{\circ} / \sqrt{\mathrm{hr}}$ \\
\hline \hline
\end{tabular}

Table 1. Performance characteristics of C-MIGITS and SPAN-LCI

\begin{tabular}{|c|c|}
\hline & VLP-16 \\
\hline $\begin{array}{c}\text { Max.Measurement } \\
\text { Range }\end{array}$ & $100 \mathrm{~m}$ \\
\hline Accuracy & $\pm 3 \mathrm{~cm}$ (typical) \\
\hline Field of view (vertical) & $30^{\circ}\left(+15^{\circ}\right.$ to $\left.-15^{\circ}\right)$ \\
\hline $\begin{array}{c}\text { Field of view } \\
\text { (horizontal) }\end{array}$ & $360^{\circ}$ \\
\hline Angular resolution & $2^{\circ} / 0.1^{\circ}$ to $0.4^{\circ}$ \\
\hline
\end{tabular}

Table 2. Performance characteristics of Velodyne LiDAR

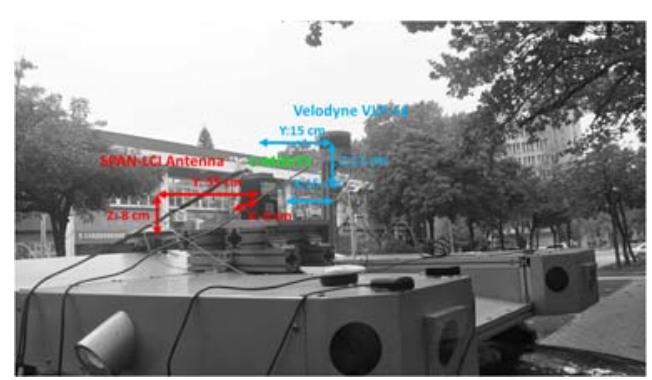

Figure 5. Configuration of the designed vehicle

Figure 6 illustrates the testing filed, Hai-an underground parking lot. It is a rectangular parking lot with two narrow central aisles. During this experiment, the indoor traveled distance in this scenario is around 1.5 kilometers and the traveled time is over 7 minutes. The scanning area in this parking lot is limited because the scanning view is blocked by walls or construction. Under this condition, SLAM suffers from the drift problem over the traveled distance. In addition, this testing field also results in the significant drift for INS-only solution since there is the less dynamic motion of the vehicle when the vehicle goes through two long aisles.

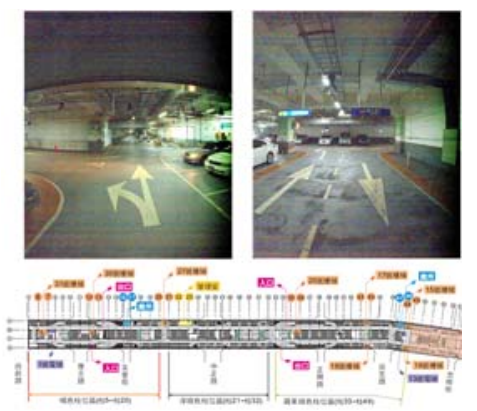

Figure 6. Overview of Hai-an underground parking lot 


\section{RESULTS AND DISCUSSIONS}

As mentioned in the experiment section, this paper utilizes commercial product and software to generate the ground truth. This ground truth is compared with the proposed method, conventional INS/GNSS and pure SLAM results. As shown in Figure 7, reference is the red trajectory from SPAN-LCI, the green line is the proposed method, the blue line represents the conventional INS/GNSS, the black line shows the result from pure SLAM and the DGNSS solution is represented as the orange dot. The GNSS-denied area can be seen from the distribution of orange dot. It is clear that blue and black line deviate from the reference while the proposed method maintains the accuracy during the whole trajectory. The maximum errors in north and east directions are over 3 meters and are indicated as black and cyan circles. Figure 7(b) illustrates the area of the maximum error where is close to the end of the indoor period. In other words, the proposed method almost keeps the positioning performance being under the 3 meters during the whole indoor period.

On the other hand, SLAM result can be interpreted as perfect in this experiment if you only take the loop closure error into account. From Figure7(b), the last position in SLAM result is very close to the reference. It means that the SLAM perfectly conduct the loop closure detection and registration. However, if we take a deep analysis in the whole trajectory, it is clear to find the trajectory also suffers from the drifting problem. By combining the INS/GNSS, this paper employs the INS-aided method to control the error in LiDAR odometer process.

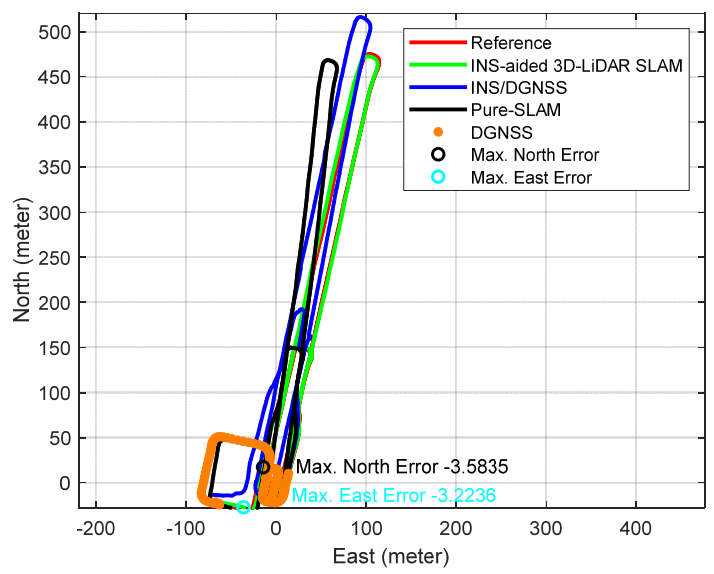

(a) whole trajectories

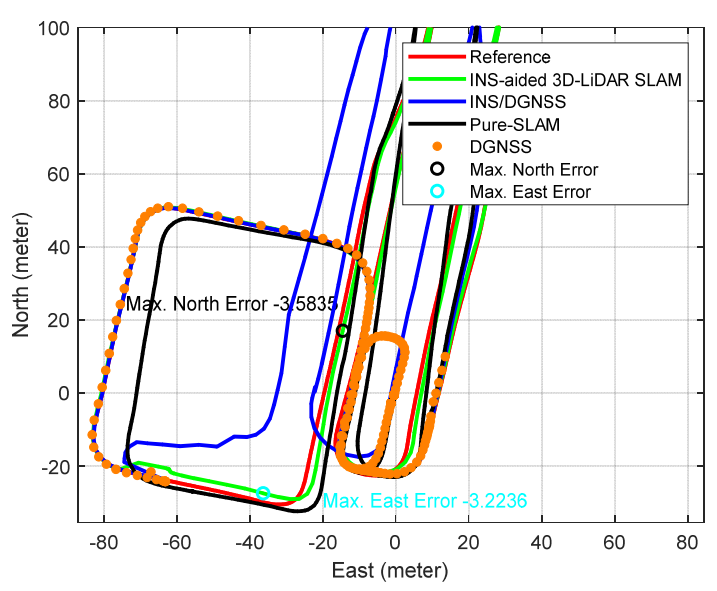

(b) zoomed trajectories

Figure 7. Trajectories of different results
Table 3 gives the overall performance of the proposed INS-aided SLAM. The RMSEs in three directions are around 1 meter. The maximum errors in three directions are all under 4 meters. Figure 8 gives the error cumulative distribution function (CDF). In Figure 8 , the proposed method can achieve meter-level accuracy in $60 \%, 2$-meter accuracy in $80 \%$ and $90 \%$ for 3-meter accuracy.

\begin{tabular}{|l|c|c|c|c|}
\hline & \multicolumn{4}{|c|}{ INS-aided LiDAR-SLAM } \\
\hline & Mean & STD & RMSE & Max. \\
\hline North (meter) & -0.007 & 1.046 & 1.047 & 3.584 \\
\hline East (meter) & 0.033 & 0.862 & 0.863 & 3.223 \\
\hline Height (meter) & -0.271 & 1.053 & 1.087 & 3.012 \\
\hline
\end{tabular}

Table 3. Evaluation of different results

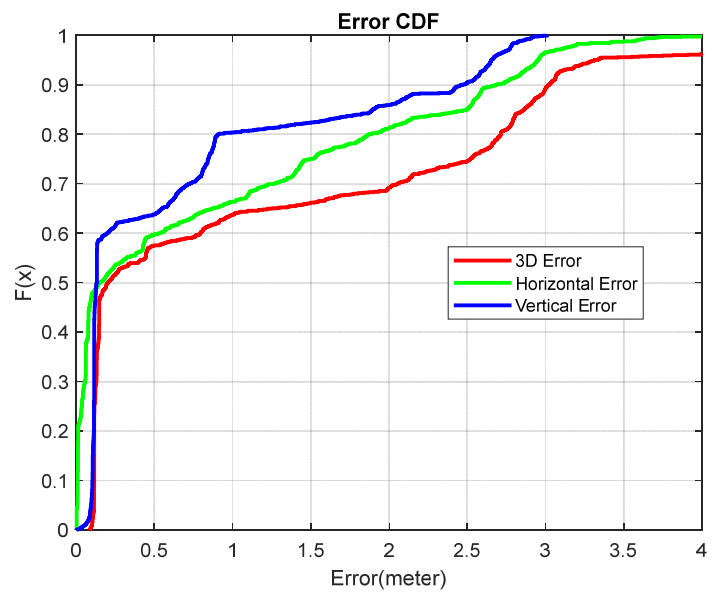

Figure 8 . The trajectories from different integration results

Figure 9 shows the point cloud information using the proposed integration method. The boundary of the underground parking lot can be perfectly matched with each scan and generate the high accuracy point cloud data. Compared with the pure SLAM, although it can also match each point cloud tightly. The result from the trajectory in Figure 7 implies the error in positioning performance which also results in the mapping performance. Moreover, the whole point cloud set is located in the global coordinate system (WGS84) by combining INS/GNSS. In other words, this point cloud can be used and shared with other system and is able to combine with the existing map or data seamlessly and efficiently.
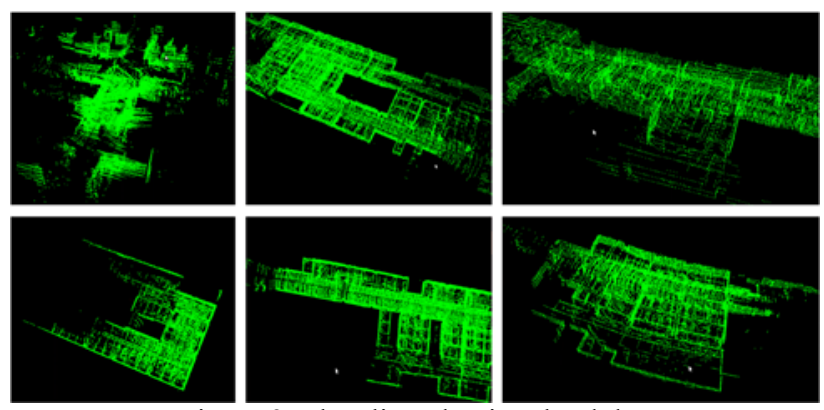

Figure 9. The aligned point cloud data

\section{CONCLUSIONS}

Multi-sensor integration is necessary for future autonomous driving. This paper proposes the fusion strategy to enhance the performance compared with the conventional methods. This paper uses information from INS/GNSS trying to solve the problems in an individual system. In the proposed structure, the 
failure solution in pure SLAM can be detected and replace with a reliable solution from INS/GNSS. By using this strategy, the drifting error significantly reduces. In addition, the consistency of the generated is improved and located in the global coordinate frame. It means this kind of map can easily and seamlessly share with other applications. The results show that the proposed method is able to achieve the meter-level accuracy even in longterm GNSS-denied environment. However, this work still needs to be enhanced. In order to meet the sub-meter accuracy in HD map, it is necessary to involve other sensors together for mitigating the error drifting or use the tightly coupled integration to improve the robustness of an integrated system.

\section{ACKNOWLEDGEMENTS}

The authors would like to thank the GEOSAT Aerospace \& Technology Inc. for its assistance in developing the land-vehicle. This work was supported by the Ministry of Science and Technology through the Project under Grant MOST 107-2221-E006-125-MY3 and 108-2917-I-006-005.

\section{REFERENCES}

Aggarwal, P., Syed, Z., Noureldin, A., El-Sheimy, N., 2010. MEMS-based integrated navigation. Artech House.

Baltsavias, E.P., 1999. Airborne laser scanning: basic relations and formulas. ISPRS Journal of photogrammetry and remote sensing, 54, 199-214.

Castorena, J., Agarwal, S., 2017. Ground-edge-based LIDAR localization without a reflectivity calibration for autonomous driving. IEEE Robotics Automation Letters, 3, 344-351.

Farrell, J., 2008. Aided navigation: GPS with high rate sensors. McGraw-Hill, Inc.

Farrell, J.A., Todd, M., Barth, M., 2016. Best practices for surveying and mapping roadways and intersections for connected vehicle applications.

Gebre-Egziabher, D., Gleason, S., 2009. GNSS applications and methods. Artech House.

Grisetti, G., Stachniss, C., Burgard, W., 2007. Improved techniques for grid mapping with rao-blackwellized particle filters. IEEE transactions on Robotics, 23, 34-46.

Hartley, R., Zisserman, A., 2003. Multiple view geometry in computer vision. Cambridge university press.

Hata, A.Y., Wolf, D.F., 2015. Feature detection for vehicle localization in urban environments using a multilayer LIDAR. IEEE Transactions on Intelligent Transportation Systems,17, 420-429.

Holz, D., Ichim, A.E., Tombari, F., Rusu, R.B., Behnke, S., 2015. Registration with the point cloud library: A modular framework for aligning in 3-D. IEEE Robotics \& Automation Magazine 22, $110-124$.

Katzenbeisser, R., 2003. About the calibration of lidar sensors, ISPRS Workshop, 1-6.

Kohlbrecher, S., Von Stryk, O., Meyer, J., Klingauf, U., 2011. A flexible and scalable slam system with full $3 \mathrm{~d}$ motion estimation, Safety, Security, and Rescue Robotics (SSRR), 2011 IEEE International Symposium on. IEEE, 155-160.
Levinson, J., Montemerlo, M., Thrun, S., 2007. Map-based precision vehicle localization in urban environments. Robotics: Science and Systems. Citeseer, 1.

Ma, L., 2017. Generation of Horizontally Curved Driving Lines for Autonomous Vehicles Using Mobile Laser Scanning Data. University of Waterloo.

Puente, I., González-Jorge, H., Arias, P., Armesto, J., 2012. Land-Based Mobile Laser Scanning Systems: Areview. Int. Arch. Photogramm. Remote Sens. Spatial Inf. Sci., XXXVIII-5/W12, 163-168.

Schenk, T., 2001. Modeling and analyzing systematic errors in airborne laser scanners.Technical Notes in Photogrammetry, 19, 46.

Seif, H.G., Hu, X., 2016. Autonomous driving in the iCity-HD maps as a key challenge of the automotive industry.Engineering , 2, 159-162.

Shin, E.-H., 2005. Estimation techniques for low-cost inertial navigation. UCGE report 20219.

Zhang, J., Singh, S., 2017. Low-drift and real-time lidar odometry and mapping. Autonomous Robots, 41, 401-416. 